\title{
Importance of Regression Analysis in Sports Information Systems at Evaluation of Sports and Sports Associations
}

ISSN: 2577-1914

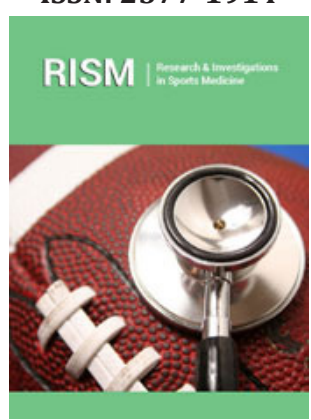

*Corresponding author: Josip Miočić, Department of Culture and Communication Sciences, Croatia

\section{Submission: 非 August 27, 2019}

Published: 侮 November 21, 2019

Volume 5 - Issue 5

How to cite this article: Josip Miočić, Korona LZ. Importance of Regression Analysis in Sports Information Systems at Evaluation of Sports and Sports Associations. Res Inves Sports Med. 5(5). RISM.000621.2019.

DOI: 10.31031/RISM.2019.05.000621

Copyright@ Josip Miočić, This article is distributed under the terms of the Creative Commons Attribution 4.0 International License, which permits unrestricted use and redistribution provided that the original author and source are credited.

\author{
Josip Miočić1* and Korona LZ² \\ ${ }^{1}$ Department of Culture and Communication Sciences, Croatia \\ ${ }^{2}$ Faculty of Philosophy, Croatia
}

\begin{abstract}
For the purpose of examining the satisfaction of sports staff with the information system supporting the evaluation of sports and sports associations, the survey technique was used, and an appropriate questionnaire was created. A survey questionnaire was filled by representatives of 93 out of 97 sports associations who applied for the Program of Public Needs in Sports in the Town of Zadar regarding the Program area of training and athlete competitions in the years of 2014 and 2015. For the purpose of this paper the regression analysis was carried out with the aim of determining the influence of individual elements as the predictors of the overall comprehensibility of the criteria for evaluating sports and sports associations. The regression analysis is described as a statistical procedure for estimating the relationship among variables. As the aim of this paper is to determine the relationship among variables, to determine the statistical dependence and the indicators of the strength of such dependence, the regression analysis technique was used as it is based on a regression model that expresses analytically the relationship between the phenomena
\end{abstract}

Keywords: Information systems; Questionnaire; Regression analysis; Predictors; Evaluation; Sports associations

\section{Introduction}

The Program of Public Needs in Sports (PPNS in further text) is of social important on all levels of the society and it is a necessity to bring it into conformity with the contemporary trends and achievements in that field in order to contribute to a more quality and more rightful evaluation of sports and sports associations as a whole through given criteria, standards and procedures. The purpose of the paper was to analyze the efficacy of the existing information system (IS in further text) within a local sports community, namely within Sports Union of the Town of Zadar and, based on the results, to propose a more efficient IS. For that purpose, content analysis, survey, in-depth interview and the regression analysis techniques were used. The objective of the paper therefore mirrors in the importance of the regression analysis and in the level of influence of elements of the given evaluation criteria as they are the predictors of the overall comprehensibility of the criteria of evaluation of sports and sports associations in terms of PPNS. As the objective of the paper was to define the relation among the variables, i.e. to determine the statistical dependence of the indicators of the strength of the dependence, the regression analysis technique was used since the technique analytically expresses the relation among phenomena.

\section{Problem}

\section{Program of public needs in sports}

The passing of the Act on Associations in the June of 2014 marked the beginning of creating a new framework of norms of functioning and financing of associations thus comprising as well sports and sports associations in Croatia because one of the two basic purposes of passing the Act was to create perquisites for an effective financing of projects and programs of common good implemented by associations in the Republic of Croatia. The Criterion, Standards and Procedures Regulation on financing and stipulating programs and projects of interest in terms of common good implemented by the associations of the Government of the Republic of Croatia decrees the basic standards and stages of financing programs or 
projects on the basic level including the sports associations in the Republic of Croatia. By means of the Regulation, the legislator established the criteria, standards and procedures to be obeyed by an entity providing funds from public sources for financing and stipulating programs to be implemented by associations including the sports ones. The Act on Associations fully and clearly defines what is considered to be programs and projects of common good implemented by associations and thus defines the activities that are in line with the values prescribed by the Constitution of the Republic of Croatia and these activities are- in a long run or within a limited time span- to give a visible added community value that improves the life of an individual and improves the development of the wider community.

Bakran etal. [1] state that the Regulation has to be as well obeyed by all and any entity co-financing or taking part in financing the common good projects on both the state and the local government levels. In line with the provisions of Article 75 of the Act on Sports, the financing of public needs in sports on the national level is done as follows: PPNS is ratified by the Croatian Parliament upon the proposal given by the Government of the Republic of Croatia and the Ministry in charge allocates funds to the Central State Office for Sports, (CSOS, since 2017), to the Croatian Olympic Committee and to other roof organizations; the roof sports associations in turn propose the programs of public needs under their competence and the programs and the financial plans are forwarded to the Ministry in charge or to the CSOS. As to bringing general acts into conformity with the Regulation when local government units are concerned, it is important to take care of the peculiarities of financing public needs on the local level and the roles of, for example, cultural councils, county and municipal sports associations etc. Based on the passed Regulation, a guidelines-structured handbook was printed, and its objective is establishing a rightful allocation of funds intended for various programs and projects of interest for common good in the Republic of Croatia including the local-level PPNS.

\section{Local-level program of public needs in sports / town of Zadar}

The case study concerning the Sports Union of the Town of Zadar (SUTZ in further text) presented a local-level PPNS. The research referred to the years of 2014 and 2015. In line with the Law on Local and Regional Self-Government and other laws and legal acts, despite the fact that a larger portion of funds intended for sports in Croatia stems from the local budgets, there is no legal obligation upon the units of local self-government concerning detailed reports on allocating the funds. The roles of counties, towns and municipalities in financing sports are determined by the legal framework and the respective normative acts. Pursuant to Article 19 of the Law on Local and Regional Self-Government, the local and regional self-government units (LSGU in further text) perform the works of local importance and through which the needs of the citizens are satisfied directly; neither the Constitution nor laws make these works a responsibility of the state bodies and, among other realms, they foremostly comprise culture, physical education and sports.
Each LSGU independently defines priorities and the strategy of the development of sports in its territory, so both PPNS and the budget funds to be allocated to it are determined in the yearly budget upon the proposal from the Sports Association. PPNS of the Town of Zadar is under authority of SUTZ and represents the basic yearly act upon which the tasks in line with the provisions of Article 76 of the Act on Sports and Article 10 of the Statute of SUTZ are performed. SUTZ integrates and proposes PPNS which is, in turn and along with the yearly budget, ratified by the Town of Zadar (the Town Council of Zadar) through the respective procedure. The starting points in creating PPNS are the evaluation of former years' performance, the legislative, structural and organizational contexts of PPNS its members, material, professional and logistic norms of sports in the town of Zadar and the status and performance on the level of the Croatian sports community. The right to take part in the Program is granted to any legal or physical person with site/ residence in the Town of Zadar and whose activities in sports can be performed based on provisions of the Act on Sports.

\section{Given criteria of evaluating sports and sports associations on local level}

SUTZ, being an element of the sports system as a whole is in charge of PPNS of the Town of Zadar and has embedded numerous factors in it, namely the binding rules of being integrated in the national organization of the system of sports, the needs and instructions of the Ministry, the proposals from the Croatian Olympic Committee and from the Town Office for Sports and Culture and recommendations from managing bodies and the SUTZ expert service. By means of acting in line with the Ordinance and obeying provisions of the Law at creating and implementing the Program, the prerequisite is provided for attaining transparency and rightfulness in allocating funds and exercising other rights resulting from the Program. The programs in the considered period were based on two legal acts passed y SUTZ: the document Criteria for Evaluation and Selection of Public Needs in Sport Program of the Town of Zadar (for the needs of evaluating sports) and the Ordinance on the Town of Zadar Sports Quality Holder (for the needs of evaluating sports association).

The evaluation criteria are general guidelines that do not have measurable nature, while the indicators deriving from them include specific measurable sizes. In the Town of Zadar, in the mentioned period, the SUTZ expert service established three basic criteria for evaluating sports with relevant indicators. These are: sport development, public sport status and sports quality. In this way, the division of sports, i.e. the status table of sports, does not arise from the notion of valuable and less valuable sports (every sport is most valuable to the one that deals with it), but from the real need for objective monitoring of the development of sports both in the town of Zadar and a wider area too. From the aforementioned, it is evident that the development of sports is a criterion, whereas the number of sports association members is an indicator that derives from the given criterion. The criterion of sport development is based on its representation at the international, national, county and city levels. 
The indicators are as follows: the number of members in the international federation, the number of members of the national alliance, the number of members of the county alliance and the number of members in the Town of Zadar. The criterion of public sport status is made up of the following indicators: the public's interest, the sport's tradition in the Town of Zadar, the Olympic sports status and the importance of teaching physical and health culture. The criterion of sporting quality refers to sports results of athletes in the Town of Zadar, and is divided into the following indicators: number of athletes categorized, achieved sports results in the last 4 years in Croatia, achieved sports results in the last 4 years in the Town of Zadar.

The "evaluation criteria", most of which are in fact the indicators of the information system for the validation of sports associations in the PPNS of the Town of Zadar, according to the Ordinance on the quality holder of the Zadar sport are: the number of registered clubs in the national league, the status of sports, the age categories in the competition the competition of the association, the number of registered members of the age categories competing, the number of members in the sports schools of the association, the professional staff and the sport tradition. In order to meet the criteria, set out by the given criteria and the attached indicators, the Association has an appropriate number of points, which determines the status of the quality bearer in each sport. In a sport, if they meet the requirements of the Rulebook, for a Qualifying Holder is determined only for one club in men's and women's competitions. Evaluation and determination of quality holders is the basis for the allocation of funds that are planned by the Town Budget based on the PPNS of the Town of Zadar [2,3].

\section{Information systems in sports for evaluation purposes}

The sport system is not a closed system, it is conceived as a segment of an integral information system of sports that connects all other information subsystems. IS collects, stores, keeps, processes and delivers information important for the organization and successful performance of all sports components, so that they are accessible to communication and usable by all players in the sport. It should be emphasized that IS can, but not necessarily, use information technology, and as such is a constituent part of the target-oriented system, whose basic function is the permanent supply of necessary information at all levels of management and decision-making at any given organizational or technological level. The contemporary IS model relies on the Internet technology, information and partnerships between sports entities and information centers that unites and channels all of this information. Milanović et al. [4] state that it is necessary to access the formulation of databases with information about characteristics and selection of athletes, sports schools, trainers and other professional qualifications, categorization, methodology and programming of sports preparation and training process etc. Accordingly, the future of computerization in various fields of sport or kinesiology is the production and use of expert systems. After the construction of a single National IS, the need for its profiling at the level of local sports communities will be assumed that will take into account the local specificities of the PPNS.

\section{Techniques and Results Analysis}

\section{Survey questionnaire}

For the purpose of testing the satisfaction of sporting staff with IS for the support of sports and sports associations, the survey technique was used, and an appropriate questionnaire was created. The survey is a special form of research through which various attitudes and opinions of authorized persons for the representation and representation of sports associations in the public are sought. The survey is the most commonly used data collection technique in social research and is particularly suitable for descriptive and causal research. It is a quantitative type of method that was conducted in the research on a sample of the entire SUTZ membership covered by the research topic.

The questionnaire survey used a survey questionnaire as an instrument of research completed by the staff of sports associations who applied to the PPNS of Zadar for the program area of training and athlete competitions in 2014 and 2015. A survey questionnaire was filled by representatives of 93 out of 97 sports associations. A good response to the survey in the implementation of the survey questionnaire is the result of the high motivation of sports association employees who are in their desire for a fairer evaluation process on the basis of established criteria and established indicators within IS. The survey questionnaire examined attitudes of sports officials authorized to represent and present sports associations on the existing IS, the given criteria and the associated indicators evaluation for the needs of PPNS of the Town of Zadar [5-9].

\section{Regression analysis}

As the aim of this paper was to establish the relationship between variables, i.e. to determine statistical dependence and the indicators of the strength of such dependence, a regression analysis was used, which analytically expresses the relation between the phenomena. More specifically, regression analysis helps to understand how the value of a dependent variable changes when any independent variables vary, while other independent variables are fixed. Most commonly, in practice, the regression analysis estimates the probable expectation of the dependent variables with respect to the independent variables, that is, the average value of the dependent variable when the independent variables are fixed. Targeted estimation is the function of independent variables, i.e. regression function. In the regression analysis it is important to characterize variations of the dependent variable around the regression function, which can be described by probability distribution. Regression analysis can be used for a variety of purposes, for example, Wong [10] used it in her research on establishing relationship on Facebook. According to Lulić [11], who states that regression analysis is often used for forecasting and forecasting, it is also used to understand the relationship between 
independent variables and to explore the forms of these relations. Under certain circumstances, the regression analysis can be used to infer the causal relationships between independent and dependent variables. However, this can lead to erroneous or false relationships because correlation does not imply causality, so caution is desirable [12].

In accordance with the afore mentioned statistical methods are included in analytical procedures, hence the use of statistical methods in the business process and functioning of sports organizations of great importance. Statistical methods facilitate the process of analyzing the occurrences and processes that have already occurred and are important for the afore mentioned sports organizations. The above methods allow for the forecasting of the same phenomena and processes based on analyzed data. Specifically, for the purposes of research, the regression analysis was carried out with the aim of determining the influence of individual elements as a predictor of the overall understanding of the criteria for evaluating the sports and the influence of individual elements as a predictor of overall understanding of the criteria for evaluation of sports associations.

\section{Results of Regression Analysis in Research}

The research results show that the afore mentioned criteria for sport valuation: sports development, public status and sports quality explain $48.7 \%$ variations in overall comprehensibility of sports evaluation criteria $(R 2=0.487, F(89)=29.156, p=0.000)$. Furthermore, sports development $(\beta=0.369, p=0.000)$ and the public status of sports $(\beta=0.405, p=0.000)$ are significant positive predictors of overall comprehension, while sports quality shows no significant contribution $(\beta=0.078, p=0.370)$ (Table 1). For the purposes of research, similarly, the regression analysis carried out on the elements of comprehensibility of the evaluation criteria of sports associations shows that these criteria explain $56.4 \%$ variations $(R 2=0.564, F(85)=16.706, p=0.000)$, where " $(\beta=0.360$, $\mathrm{p}=0.000)$ and "professional staff" $(\beta=0.311, \mathrm{p}=0.001)$ show significant predictors while others do not show significant impact on overall comprehensibility (Table 2).

Table 1: Regression analysis of the influence of individual elements as a predictor of overall comprehensibility of the criteria for the evaluation of sports.

\begin{tabular}{|c|c|c|c|c|}
\hline & B & Beta & T & Sig. \\
\hline Sports development &, 382 &, 369 & 3,912 &, 000 \\
\hline Public status of sorts &, 384 &, 405 & 4,587 &, 000 \\
\hline Sports quality &, 094 &, 078 &, 901 &, 370 \\
\hline
\end{tabular}

Table 2: Regression analysis of the impact of individual elements as a predicator of overall comprehension of criteria for the evaluation of sports associations.

\begin{tabular}{|c|c|c|c|c|}
\hline & B & Beta & $\mathbf{T}$ & Sig. \\
\hline The number of registered clubs in the national federations & ,149 & ,256 & 3,200 & 002 \\
\hline Type / status of sport & ,184 & ,360 & 3,836 & 000 \\
\hline Representation of age categories in the competition & ,292 &,- 134 & $-1,255$ & ,213 \\
\hline Ranking of the Association's competition & -132 & ,119 & 1,105 & ,273 \\
\hline The number of registered members of the age categories of the association that compete & ,112 & 012 & 113 & 910 \\
\hline Number of members / mass in the sports schools of the Association & 011 & 168 & 1,853 & 068 \\
\hline Professional staff & 156 & 311 & 3,593 & 001 \\
\hline
\end{tabular}

\section{Conclusion}

Based on the survey conducted and the results of the questionnaire survey conducted by representatives of 93 sports associations (out of a total of 97), a series of topics emerged from questions from the survey questionnaire. The respondent's response from the questionnaire was generated from the desire or motivation for a more rightful evaluation of sports and sports associations. The evaluation process is more transparent and more appropriate as the set criteria and established indicators within IS are better, so the respondent's response is understandable and expected. In the survey, attitudes were examined, that is, the satisfaction of sports workers and authorized representatives of sports associations with the existing IS, the given criteria and the added valuation indicators for the needs of PPNS Zadar. With the results of the research, it was found that the IS to support the evaluation of sports and sports associations through PPNS is only partly satisfactory for sports workers. From the above results, it follows that most respondents are mostly or partially certain about the criteria, indicators and procedures in the IS for the promotion of sports and sports associations through the PPNS local sports community.

It was established that the evaluation criteria (52\%), indicators (54\%) and procedures (47\%) were (only) partially clear to most respondents, i.e. that only a small number of respondents were fully certain (evaluation criteria - 26\%, indicators) \%, procedures - 40\%). The results also showed that there is a partial lack of understanding (or misunderstanding) of the process of evaluating sports and sports associations through PPNS. As a result of the research, it was discovered that most of the respondents evaluated sports and sports associations through PPNS as completely or 
partially comprehensible (74\%). On the other hand, $41.9 \%$ of the respondents argued that there is generally some misunderstanding, and $43 \%$ of respondents have a general misunderstanding of the process of evaluating sports and sports associations through PPNS. Therefore, we can conclude that there is a misinterpretation (or misinformation) of the process of valuation of sports and sports associations of sports workers of the local sports community. It should be noted once again that in the majority of questions about lack of understanding (or misunderstanding), the answer was boiled down to "partially", which ultimately means that there is only a partial lack of understanding (or misunderstanding) of the evaluation process of sports and sports associations through the PPNS. In order to facilitate the process of analyzing the occurrences and processes that are important for the aforementioned sports organizations, a regression analysis was carried out for the purposes of research. This analysis identified the impact of individual elements as a predictor of the overall understanding of the criteria for evaluating sports and sports associations. The results show that the criteria of "sport development" and "public status of sport" represent significant predictors of overall comprehension, while sporting quality as such does not show a significant contribution. Similarly, regression analysis conducted on elements of the comprehensibility criteria of sports associations has shown that these criteria explain $56.4 \%$ variation, with professional staff, sport / sport status and number of registered sports clubs as significant predictors, while the other evaluation criteria do not show a significant impact on the overall understanding of the criterion of evaluation of sports associations.

\section{References}

1. Bakran D, Cutvaric M, Jakir BI, Karacic M, Lendic KV, et al. (2016) Financial operations of non-profit organizations. RiF, Zagreb, p. 605.

2. Bronić M (2012) Financing sport in the Republic of Croatia with a comparative overview of financing in the European Union: A research project. Institute of Public Finance, Zagreb, Croatia.

3. Miocic J (2018) Modeling information systems to support evaluation processes in sport. Doctoral dissertation, University of Zadar, Croatia.

4. Milanovic D, Custonja Z, Bilic D (2011) Basic principles and guidelines for the development of sports in the Republic of Croatia. Zagreb, Croatia.

5. Republic of Croatia (2006) Sports Act. Official Gazette, 71/06 (150/8, $124 / 10,124 / 11,86 / 12,94 / 13,85 / 15,19 / 16,98 / 19)$.

6. (2011) Zadar Sports Association.

7. Stambuk A, August BM (2013) Regression and correlation analysis.

8. Vercic AT, Sincic D, Vokic NP (2010) Handbook of research methodology: How to design, conduct, and describe scientific and professional research. Zagreb, Croatia.

9. Vogt $\mathrm{H}$ (2004) Putting the customer first! managing customer satisfaction. Bertelsmann Stiftung, Gütersloh, Germany.

10. Wong WKW (2012) Faces on facebook: A study of self-presentation and social support on facebook. SS Student E-Journal 1: 184-214.

11. Lulic I (2014) Use of regression analysis method in solving problems related to engineering practice. Doctoral dissertation, Faculty of Mechanical Engineering and Naval Architecture, University of Zagreb, Croatia.

12. Office for Associations of the Government of the Republic of Croatia (2015) Implementation manual: Regulations on criteria, benchmarks and procedures for financing and contracting programs and projects of common interest. Zagreb, Croatia.

For possible submissions Click below: 